\title{
Juvenile experience alters strategies used to solve the radial arm maze in rats
}

\author{
JEREMY L. HALL and ROBERT F. BERMAN \\ Wayne State University, Detroit, Michigan
}

\begin{abstract}
Previous research demonstrated that juvenile rats solve the radial arm maze by selecting adjacent arms, whereas adult rats use a less restrictive response strategy. In the present experiment, these findings were replicated and extended to include a third group of rats tested both as juveniles and as adults, and these response patterns were compared in two strains of rats. Radial eight-arm maze performance was assessed daily for 10 days in juvenile and adult Long Evans and Sprague-Dawley rats. In both strains, juveniles made significantly more adjacent arm entries than did adults. In addition, both strains of rats, when retested as adults, continued to use the adjacent arm entry strategy established during juvenile training, rather than adopting the maze-naive adult strategy. Together, these results support previous findings demonstrating that juvenile rats use an adjacent arm entry strategy to solve the radial eight-arm maze, whereas adult rats do not. In addition, these findings suggest that juvenile maze experience produces a long-lasting change in the response patterns of rats in the radial eight-arm maze.
\end{abstract}

Radial arm maze performance is routinely used to assess learning and memory processes. This task requires animals to enter each arm of a radial maze for a food reward without revisiting arms (Olton \& Samuelson, 1976). There are several possible ways to solve the maze, including the use of extramaze cues, intramaze cues, or simple response strategies. These response strategies have come to be known by several names, including kinesthetic strategy (Pico \& Davis, 1984), nonspatial strategy (Watts, Stevens, \& Robinson, 1981), and egocentric localizing strategy (O'Keefe \& Nadel, 1978).

Previous studies demonstrated that the techniques used to solve the radial arm maze are dependent on genetic, developmental, experiential, and contextual factors. For example, Rossi-Arnaud, Fagioli, and AmmassariTeule (1991) reported differing performance levels and strategies used by $\mathrm{C} 57 \mathrm{BL} / 6$ and DBA/2 mice. Radial arm maze strategies are also dependent on gender; female rats are less likely than male rats to use an adjacent arm entry strategy (Einon, 1980). The age of animals also appears to be an important determining factor in strategy selection. Juvenile animals utilize an adjacent arm entry strategy, whereas adult animals do not (Einon, Will, \& Morgan, 1980). Similarly, rats raised in an isolated environment are slower to adopt the adjacent arm entry strategy than are animals raised in a social environment (Einon, 1980)suggesting that experiential factors may affect radial arm maze strategies.

This research was supported by National Institute of Alcohol and Alcohol Abuse Grant P50AA07606. Correspondence concerning this article should be addressed to J. L. Hall, Department of Psychology, 71 W. Warren, Wayne State University, Detroit, MI 48201 (e-mail: jhall@gopher.chem.wayne.edu).
The purpose of the following study was to evaluate the possibility that radial arm maze experience in juvenile animals might alter the technique used to solve the maze in early adulthood, and to compare radial arm maze performance in two strains of rats.

\section{METHOD}

\section{Animals}

Ninety-day-old nulliparous female and 75-day-old male SpragueDawley and Long-Evans rats (Harlan Labs; Portage, MI) were singly housed in an environmentally controlled vivarium, maintained at $22^{\circ} \pm 1^{\circ} \mathrm{C}$ with lights on at $0700 \mathrm{~h}$ and off at $1900 \mathrm{~h}$. The animals had ad-lib access to lab chow and water for 2 weeks prior to beginning the experiment.

Each female was mated to a randomly assigned male. Mating occurred in each female's cage for $4 \mathrm{~h}$ daily and continued for 2 weeks or until a vaginal plug was found. Sperm-positive females were singly housed until parturition. At birth, offspring were counted, weighed, randomly culled to 10 per litter, and returned to the dams. Offspring were weighed and weaned at 21 days of age, separated by gender, and group housed (3-4 per cage) prior to behavioral testing.

Two cohorts of male pups from each strain were used $(n \mathrm{~s}=8)$. The juvenile cohort was singly housed, placed on a restricted feeding schedule sufficient to ensure continued growth (5-15 g Purina Rat Chow/day), and subjected to behavioral training beginning on Postnatal Day 26. Following radial arm maze testing (see below), these pups were maintained in single housing and given ad-lib access to lab chow until 85 days of age, at which time food intake was again restricted, this time to a level that maintained body weights at $80 \%$ of free-feeding weights. On Postnatal Day 90, radial arm maze performance of these animals was reassessed (retest) as before, except that no familiarization trials were provided. Animals in the adult cohort were singly housed on Postnatal Day 75 , food deprived to $80 \%$ of free-feeding weights, and then familiarized and trained in the radial arm maze beginning on Postnatal Day 90. One Long-Evans rat from the adult cohort was eliminated for remaining virtually immobile in the maze throughout the 15 days of exposure. 


\section{Apparatus}

The floor of the maze was plywood, painted white. The center of the maze was octagonal ( $25 \mathrm{~cm}$ side to side) and was surrounded by clear Plexiglas $30.5 \mathrm{~cm}$ high. Each of the eight arms $(55.5 \times$ $7.1 \mathrm{~cm}$ ) projected from the center of the maze and had clear Plexiglas walls ( $15.6 \mathrm{~cm}$ high). The entrance to each of the arms was blocked by a clear Plexiglas guillotine door remotely controlled via overhead lines. Recessed cups were located at the end of each arm $(2.5 \mathrm{~cm}$ diameter $\times 1.8 \mathrm{~cm}$ deep). The maze was constructed on a pedestal $(75 \mathrm{~cm}$ high) in a dimly lit room with several large extramaze cues present (e.g., doors, chalkboards, experimenter). The maze was cleaned daily prior to training.

\section{Behavioral Procedures}

All behavioral procedures were conducted between $1000 \mathrm{~h}$ and $1700 \mathrm{~h}$. The animals were familiarized with the maze by placing each animal in the center, simultaneously opening the guillotine doors, and allowing the animals to explore the unbaited maze ( $5 \mathrm{~min} / \mathrm{trial}, 1 \mathrm{trial} /$ day, 5 days total). Following this familiarization period, the animals were trained to locate and consume food rewards (Froot Loops Cereal) located in the recessed cups at the end of each $\mathrm{arm}(5 \mathrm{~min} / \mathrm{trial}, 1 \mathrm{trial} /$ day, 10 days total $)$.

\section{Scoring}

Failure to enter an arm, reentry into a previously visited arm, or entry into an arm but failure to consume the food reward was scored as an error (Altman, Ogren, Berman, \& Normile, 1989). Entering an arm immediately adjacent to the arm being exited (either to the left or to the right) was considered an adjacent arm entry.

\section{Data Analyses}

Effects on maze performance (i.e., number of errors) and adjacent arm entries made during the first eight arm choices daily were assessed with a multivariate analysis of variance.

\section{RESULTS}

Figures 1 and 2 illustrate the number of errors made by Sprague-Dawley and Long-Evans rats, respectively,

\section{SPRAGUE-DAWLEY}

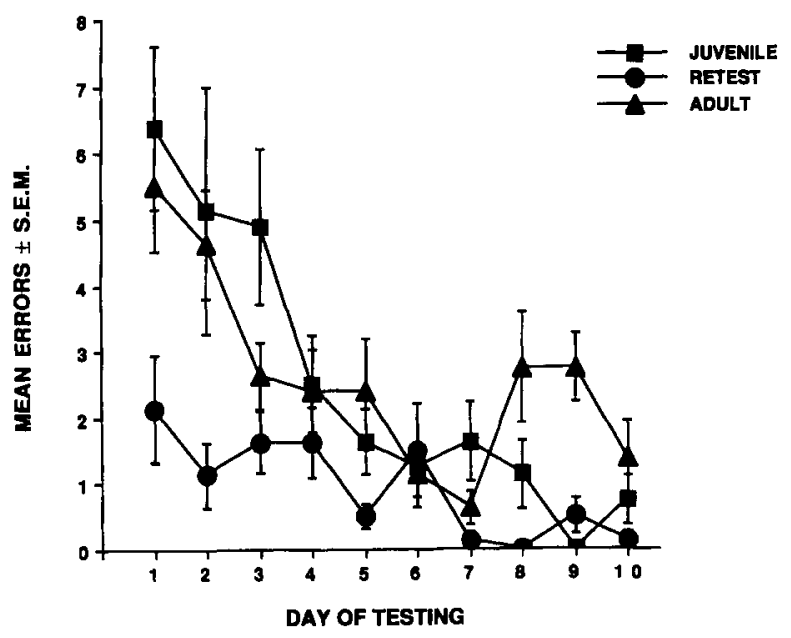

Figure 1. Radial arm maze performance in Sprague-Dawley rats. Note that animals in the retest condition made significantly fewer errors than did rats in the juvenile or adult condition (which did not substantially differ from one another), indicating substantial memory savings from previous training.
LONG EVANS

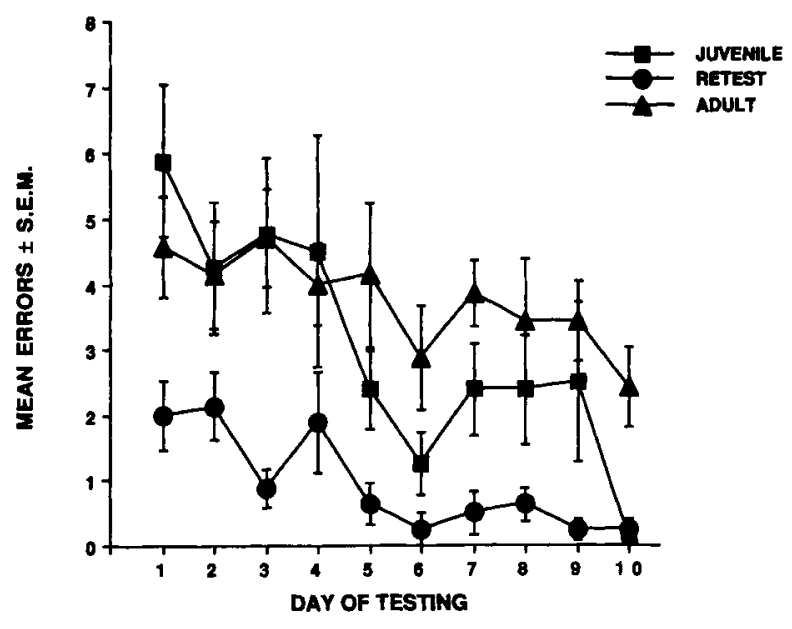

Figure 2. Radial arm maze performance in Long-Evans rats. Note that animals in the retest condition made significantly fewer errors than did rats in the juvenile or adult condition (which did not substantially differ from one another), indicating substantial memory savings from previous training.

tested in the radial arm maze. The effect of trials was significant $[F(9,369)=17.16, p<.01]$, indicating that the animals learned to solve the maze. The main effect of testing condition was also significant $[F(2,41)=23.10$, $p<.01]$, as was the interaction between trials and testing condition $[F(18,369)=2.44, p<.01]$, with animals in the retest condition making significantly fewer errors than did animals in the juvenile condition $[F(1,41)=$ $27.89, p<.01]$ or the adult condition $[F(1,41)=39.96$, $p<.01]$, indicating substantial memory savings in the retested animals from prior training. The effect of strain approached, but did not reach, statistical significance $[F(2,41)=36.31, p<.06]$.

Figures 3 and 4 illustrate the number of adjacent arm entries made by Sprague-Dawley and Long-Evans rats respectively in the maze. The effect of trials was again significant $[F(9,369)=8.56, p<.01]$, as was the interaction between trials and condition $[F(18,369)=2.11$, $p<.01]$. The main effect of testing condition was significant $[F(2,41)=15.44, p<.01]$, with juvenile $[F(1,41)=$ $28.65, p<.01]$ and retested animals $[F(1,41)=16.37$, $p<.01]$ making more adjacent arm entries than did adult naive animals, indicating that once the adjacent arm entry strategy had been adopted by juvenile animals, it continued to be used despite increasing age. There was no significant effect of strain on the number of adjacent arm entries.

\section{DISCUSSION}

Both Sprague-Dawley and Long-Evans rats used an adjacent arm entry strategy to solve the radial arm maze when initially tested as juveniles, but not when initially tested as adults. When retested as adults after prior ex- 
SPRAGUE-DAWLEY

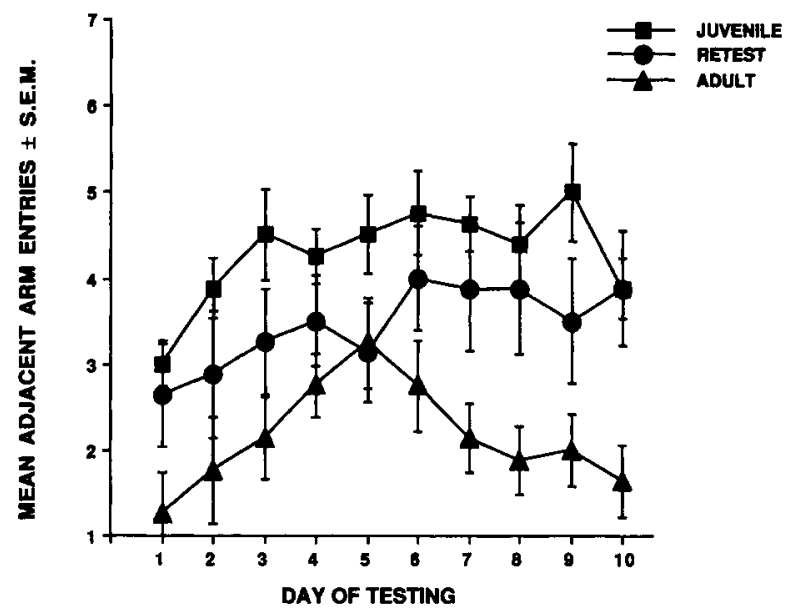

Figure 3. Adjacent arm entries made by Sprague-Dawley rats during radial arm maze testing. Note that animals in both the juvenile and retest conditions consistently relied on an adjacent arm entry strategy to solve the maze, whereas animals in the adult condition appeared to use this strategy only during the initial acquisition period (see Figure 1), after which other cues were apparently used to successfully navigate through the maze.

perience in the radial arm maze as juveniles, animals from both strains continued to use the adjacent arm entry strategy, despite an apparent age-related shift in maze technique. Both Sprague-Dawley and Long-Evans rats rapidly acquired the task, with no significant strain differences in the number of errors made in the radial arm maze in any condition, although Sprague-Dawley rats did appear to learn the maze somewhat more quickly. Thus, radial arm maze performance of Sprague-Dawley rats and that of Long-Evans rats appear to be essentially comparable.

These results support previous findings describing agerelated changes in radial arm maze strategies in rats (Einon et al., 1980). Dale and Innis (1986) reported an adjacent arm entry strategy being employed by 5 -monthold Long-Evans rats, but these rats had received 20 trials on the radial arm maze prior to beginning that experiment. Given the results of our study, it would appear that this prior maze experience probably altered the strategy used by these animals as adults. This apparent contradiction highlights the importance of understanding the effects of prior experience and of the postnatal environment on behavior.

In a longitudinal study, similar in design to our own, of radial arm maze performance in rats from 4 to 25 months of age, Caprioli and associates (Caprioli, Ghirardi, Giulani, Ramacci, \& Angelucci, 1991; Caprioli, Ghirardi, Ramacci, \& Angelucci, 1990) reported that previous adult experience did not affect the strategy used to solve the radial arm maze by aged rats (adult rats used an adjacent arm entry strategy whereas aged rats did not, regardless of prior maze experience). These apparently discrepant findings are most likely the result of an important method- ological difference. Caprioli et al. (1991; Caprioli et al., 1990) used a maze constructed of opaque Plexiglas; we used a maze constructed of translucent Plexiglas. Thus, animals in the study by Caprioli et al. (1991; Caprioli et al., 1990 ) were required to rely on intramaze or kinesthetic cues, whereas in our study the animals could also utilize extramaze cues-suggesting that in addition to genetic, developmental, and experiential factors, radial arm maze strategies also depend on the availability of contextual cues. In this regard, Dale and Innis (1986) tested blinded, adult, maze-naive rats and reported that $98.5 \%$ of the arm choices made by these rats were to adjacent arms.

Since experiential factors appear to be important in determining radial arm maze strategies (Einon, 1980), we must also approach our own findings with caution. We did not control for the effects of juvenile experience in the adult animals. The adult retest animals received daily elevator rides, handling, and maze training while they were still juveniles, whereas the adult maze-naive animals did not. Thus, a similar effect on radial arm maze strategy might have been obtained simply by placing juvenile animals in any environmentally enriching condition (e.g., open field, Morris water maze, Stone maze, etc.) and testing them at a later age in the radial arm maze.

A similar methodological concern arises when one considers the performance of the adult naive animals. Einon (1980) clearly showed that social rearing conditions produce an increased likelihood of using an adjacent arm entry strategy to solve the radial arm maze in Wistar rats. Our maze-naive adult animals were group housed until Postnatal Day 75; our retested adult animals were singly housed from Postnatal Day 26. However, such an effect should work against our hypothesis, since the retested animals ought to have been the ones not to use the adjacent arm entry strategy, whereas the adult naive would have been more likely to use it. The opposite

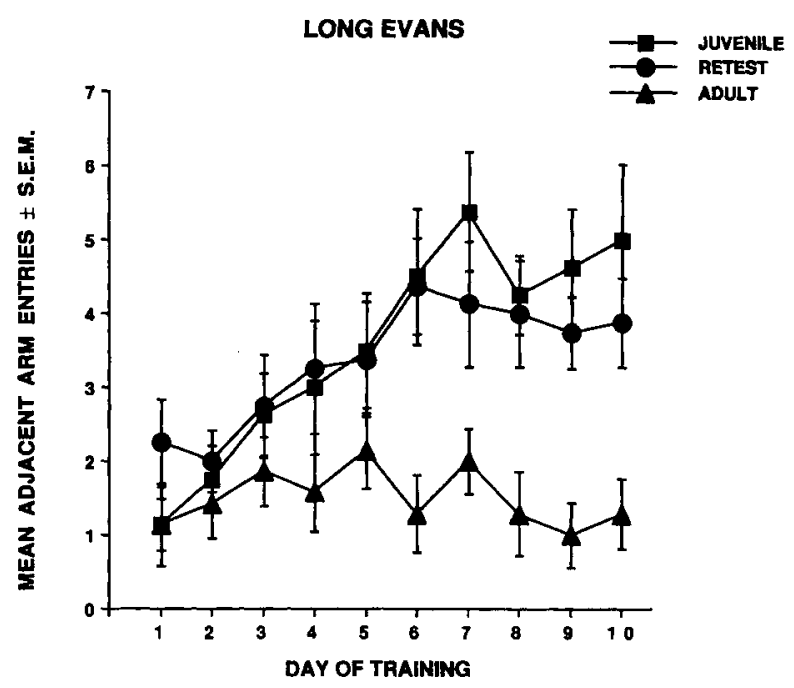

Figure 4. Adjacent arm entries made by Long-Evans rats during radial arm maze testing. Note that animals in the juvenile and retest conditions consistently relied on an adjacent arm entry strategy to solve the maze, whereas animals in the adult condition did not. 
effect was evident in our study, with the naive animals not using the strategy whereas the retested animals did.

One possible explanation of the difference in maze strategies used by naive juvenile and naive adult animals may correspond to hippocampal development. Perhaps juvenile animals used the adjacent arm entry strategy because the capacity for spatial learning (i.e., the hippocampi) had not yet fully developed, whereas in the adult naive animals, it had. Alternatively, perhaps development of the visual system was a critical factor. Since Dale and Innis (1986) showed that blinded adult animals prefer the adjacent arm entry strategy, it is possible that the reason why the juvenile animals also used the adjacent arm entry strategy was that they could not see as well as the adult animals. On the other hand, the relative size of the maze for the two age groups might have played a role in these findings. The smaller, juvenile animals had a proportionately greater distance to run to receive the Froot Loops than did the larger, adult animals and might therefore have used the adjacent arm entry strategy simply as a means of locomotor economy. In this regard, Yoerg and Kamil (1982) showed that the larger the diameter of the central platform, the more likely animals were to use an adjacent arm entry strategy. However, none of these possible explanations suggests why the retested animals continue to use the adjacent arm entry strategy after reaching full maturity. Perhaps this effect is a simple win/stay phenomenon. Once the animals have learned an efficient means of solving the maze, there is no reason to develop a new system.

\section{REFERENCES}

Altman, H. J., Ogren, W. O., Berman, R. F., \& Normile, H. J. (1989). The effects of $p$-chloroamphetamine, a depletor of brain serotonin, on the performance of rats in two types of positively reinforced com- plex spatial discrimination tasks. Behavioral \& Neural Biology, 52, 131-144.

Caprioli, A., Ghirardi, O., Giuliani, A., Ramacci, M. T., \& ANGELUCCI, L. (1991). Spatial learning and memory in the radial maze: A longitudinal study in rats from 4 to 25 months of age. Neurobiology of Aging, 12, 605-607.

Caprioli, A., Ghirardi, O., Ramacci, M. T., \& Angelucci, L. (1990). Age-dependent deficits in radial maze performance in the rat: Effect of chronic treatment with acetyl-L-carnitine. Progress in Neuropsychopharmacology \& Biological Psychiatry, 14, 359-369.

DALE, R. H., \& INNIS, N. K. (1986). Interactions between response stereotypy and memory strategies on the eight-arm radial maze. Behavioural Brain Research, 19, 17-25.

EINON, D. [F.] (1980). Spatial memory and response strategies in rats: Age, sex and rearing differences in performance. Quarterly Journal of Experimental Psychology, 32, 473-489.

Einon, D. F., WiLl, B. E., \& Morgan, M. J. (1980). Effects of postoperative environment on recovery from dorsal hippocampal lesions in young rats: Tests of spatial memory and motor transfer. Quarterly Journal of Experimental Psychology, 32, 137.

O'KeEfE, J., \& NADEL, L. (1978). The hippocampus as a cognitive map. Oxford: Oxford University Press, Clarendon Press.

Olton, D. S., \& SAmuelson, R. J. (1976). Remembrance of places past: Spatial memory in rats. Journal of Experimental Psychology: Animal Behavior Processes, 2, 97-116.

Pico, R. M., \& Davis, J. L. (1984). The radial maze performance of mice: Assessing the dimensional requirements for serial order memory in animals. Behavioral \& Neural Biology, 40, 5-26.

Rossi-Arnaud, C., Fagioli, S., \& Ammassari-Teule, M. (1991). Spatial learning in two inbred strains of mice: Genotype-dependent effect of amygdaloid and hippocampal lesions. Behavioural Brain Research, 45, 9-16.

WATTS, J., STEVENS, R., \& RoBinson, C. (1981). Effects of scopolamine on radial maze performance in rats. Physiology \& Behavior, 26, 845-851.

YoERG, S. I., \& KAMIL, A. C. (1982). Response strategies in the radial arm maze: Running around in circles. Animal Learning \& Behavior, 10, 530-534.

(Manuscript received December 6, 1994; revision accepted for publication February 27, 1995.) 